Kansas State University Libraries

New Prairie Press

\title{
THE INFORMATION-THEORETIC APPROACH TO MODEL SELECTION: DESCRIPTION AND CASE STUDY
}

Erin E. Blankenshipa

Micah W. Perkinsb

Ron J. Johnsonc

Follow this and additional works at: https://newprairiepress.org/agstatconference

Part of the Agriculture Commons, and the Applied Statistics Commons

\section{(c) (1) $\Theta(9$}

This work is licensed under a Creative Commons Attribution-Noncommercial-No Derivative Works 4.0 License.

\section{Recommended Citation}

Blankenshipa, Erin E.; Perkinsb, Micah W.; and Johnsonc, Ron J. (2002). "THE INFORMATION-THEORETIC APPROACH TO MODEL SELECTION: DESCRIPTION AND CASE STUDY," Conference on Applied Statistics in Agriculture. https://doi.org/10.4148/2475-7772.1200

This is brought to you for free and open access by the Conferences at New Prairie Press. It has been accepted for inclusion in Conference on Applied Statistics in Agriculture by an authorized administrator of New Prairie Press. For more information, please contact cads@k-state.edu. 


\title{
The Information-Theoretic Approach to Model Selection: Description and Case Study
}

\author{
Erin E. Blankenship ${ }^{a}$, Micah W. Perkins ${ }^{b}$, and Ron J. Johnson ${ }^{c}$ \\ ${ }^{a}$ Department of Biometry, University of Nebraska, Lincoln, NE 68583-0712 \\ ${ }^{b}$ Joseph W. Jones Ecological Research Center, Newton, GA 317r0-9651 \\ ${ }^{c}$ School of Natural Resource Sciences, University of Nebraska, Lincoln, NE 68583-0819
}

\begin{abstract}
In the wildlife literature there has been some recent criticism of statistical significance testing. In the past few years, both the Journal of Wildlife Management and the Wildlife Society Bulletin have published articles criticizing the overuse and misuse of hypothesis tests. One alternative to using hypothesis tests for model selection is the information-theoretic approach, proposed by Burnham and Anderson (1998). This technique uses values such as the Akaike Information Criterion and others to choose a set of plausible models from a set of a priori candidate models. Inferences are based on the set of plausible models, rather than on a single selected best model, and model-averaged point estimates of parameters may be used for prediction. The Burnham and Anderson method is gaining popularity in the wildlife science community, and statisticians who work with wildlife scientists should be aware of this analysis technique and how to use it properly. This paper will introduce statisticians to the information-theoretic approach to model selection and the statistical theory underlying it, as well as demonstrate the technique using data on bird species richness and abundance in riparian areas in southeastern Nebraska.
\end{abstract}

KEY WORDS: Akaike information criterion, Schwarz's Bayesian Information Criterion, Kullback-Liebler distance. 


\section{Applied Statistics in Agriculture}

\section{Introduction}

Recently in the wildlife literature, several articles have appeared criticizing the use of statistical significance testing. These papers, appearing in the Wildlife Society Bulletin and The Journal of Wildlife Management, focus on the overuse and misuse of hypothesis tests. Cherry (1998) counted the number of p-values that appeared in the 1995 issue of The Journal of Wildlife Management, and found more than 2,400. He uses this large number as evidence of the overuse of tests, and notes that criticism of statistical testing has appeared in many disciplines, including statistics itself. Wildlife, it seems, is merely the latest discipline to note the widespread misuse of testing. Using quotes from Frank Yates, D. R. Cox and John Nelder, Cherry (1998) presents four major problem areas that he believes has led to this proliferation of inappropriate p-values. They include unnecessary testing, confusion over power analyses, misunderstanding the assumptions of hypothesis tests, and fixed-level tests.

These are the same problem areas discussed by Johnson (1999). Johnson blames the overuse and misuse of statistical tools in the wildlife field on the increased emphasis on hypothesis testing and power analyses. He gives various incorrect ways scientists interpret p-values, and contends that the correct interpretation of the p-value depends on assumptions that are usually questionable. In particular, he questions the calculation of the p-value given a true null hypothesis. He argues that point null hypotheses are "almost invariably known to be false before any data are collected" (Johnson 1999) and this leads to "gratuitous" significance testing. Johnson does note that while point null hypotheses are nearly always false in observational studies, they are often reasonable for designed experiments.

It is the distinction between observational studies and designed experiments that is the focus of other articles in this area. Anderson et al. (2000) contend that the paradigm of significance testing is not wrong, but simply that it is not informative. They also note that

significance testing is not particularly useful in model or variable selection. The importance of model and variable selection is perhaps the most important distinction between the analyses of observation studies and designed experiments. Most wildlife studies are observational, and the analysis of such studies tends to focus on selecting the set of predictor variables which best explain the variation in the response variable. Cherry (1998) and Anderson et al. (2001) both point out that testing in regression settings tends to focus on testing 
whether a relationship between the predictor and response variable exists. The question of interest, however, is usually not whether a relationship exists, but what kind of relationship exists. Cherry (1998), Thompson (1999), and Anderson et al. (2000) all contend that this question can best be answered by estimation, rather than testing. Anderson et al. (2000) propose an alternative to null hypothesis testing that incorporates both model selection and estimation. This method, termed the information-theoretic approach, bases model selection on information criteria and estimates effects using model averaging.

The information-theoretic approach, detailed in Burnham and Anderson (1992, 1998) and Anderson et al. (2000, 2001), has been gaining popularity in the wildlife literature as an analysis method. The purpose of this paper is to introduce consulting statisticians to the information-theoretic approach. This includes both the statistical theory underlying the approach, as well as how the method can be practically used for data analysis. The use of the information-theoretic method will be demonstrated using data on bird species richness and abundance in riparian areas in southeastern Nebraska. Finally, some criticisms and drawbacks of the information-theoretic approach will be presented.

\section{The Information-Theoretic Approach: Theory}

Burnham and Anderson (1998) give a detailed description of the statistical theory behind the information theoretic method. This section presents an overview of the arguments described in Burnham and Anderson (1998).

The goal of the information-theoretic approach to model selection is finding the best model to describe the relationship between a set of predictor variables and a response variable. It is based on the concept of minimizing the distance between two models. Burnham and Anderson (1998) describe how Kullback and Leibler derived a measure of the discrepancy between two models, $f$ and $g$. This measurement, called the Kullback-Leibler (K-L) distance, measures the discrepancy between the "truth" $(f)$ and an approximating model $(g)$. The smaller the K-L distance, the closer the approximating model $g$ comes to the truth $(f)$. The approximating model $g$ depends on parameters $\theta$, and so is written $g(x \mid \theta)$. The "truth" depends only on the data, $f(x)$. The K-L distance between models $f$ and $g$ is defined 


\section{Applied Statistics in Agriculture}

as

$$
\mathrm{I}(f, g)=\int f(x) \log \left(\frac{f(x)}{g(x \mid \theta)}\right) d x
$$

where $f$ and $g$ are continuous functions. Anderson et al. (2000) define $I(f, g)$ as the "information" lost when the truth $f$ is approximated by $g$. The goal of model selection is then to minimize $\mathrm{I}(f, g)$ over $g$. If $\mathrm{I}(f, g)$ is to be minimized over $g$, then the $\mathrm{K}$-L distance must be calculated for various approximating models $g$. But, $f$ is "truth," which is unknown. To see how the K-L distance may be used with an unknown $f$, Burnham and Anderson (1998) and Anderson et al. (2000) write $\mathrm{I}(f, g)$ in a different way:

$$
\begin{aligned}
\mathrm{I}(f, g) & =\int f(x) \log \left(\frac{f(x)}{g(x \mid \theta)}\right) d x \\
& =\int f(x) \log (f(x)) d x-\int f(x) \log (g(x \mid \theta)) d x \\
& =\mathrm{E}_{f}[\log (f(x))]-\mathrm{E}_{f}[\log (g(x \mid \theta))] \\
& =C-\mathrm{E}_{f}[\log (g(x \mid \theta))] \\
\mathrm{I}(f, g)-C & =-\mathrm{E}_{f}[\log (g(x \mid \theta))]
\end{aligned}
$$

where $C$ is a constant that is fixed across all approximating models $g$. The quantity $\mathrm{I}(f, g)-C$ is the relative $\mathrm{K}-\mathrm{L}$ distance and $\mathrm{E}_{f}[\log (g(x \mid \theta))]$ is therefore the focus in model selection (Anderson et al. 2000). The basic principle of the information-theoretic approach is to postulate an a priori set of candidate models $g_{i}(x \mid \theta)$ and select the best of these as the model that minimizes $\mathrm{E}_{f}[\log (g(x \mid \theta))]$.

This quantity, $\mathrm{E}_{f}[\log (g(x \mid \theta))]$, cannot be calculated because the parameters $\theta$ are unknown. Instead, an estimate of the relative $\mathrm{K}-\mathrm{L}$ distance between $f$ and $g_{i}$ would help select the best of the candidate models. This means that model selection could be based on minimizing the expected relative K-L distance (Anderson et al. 2000).

Burnham and Anderson (1998) describe how Akaike, in 1973, found an estimator of the expected relative $\mathrm{K}$-L distance based on a bias corrected maximized log-likelihood value-the Akaike Information Criterion (AIC):

$$
\mathrm{AIC}=-2 \log (l(\widehat{\theta}))+2 p
$$


where $p$ is the number of estimated parameters in the approximating model, $g$. The approximating model with the smallest AIC value is considered to be "closest" to the truth (Burnham and Anderson 1998, Anderson et al. 2000).

Burnham and Anderson (1998) and Anderson et al. (2000, 2001) emphasize that if there are too many parameters $p$ with respect to the sample size $n$, then $\mathrm{AIC}$ will perform poorly. Instead, a modified criterion, $\mathrm{AIC}_{c}$, should be used

$$
\begin{aligned}
\mathrm{AIC}_{c} & =-2 \log (l(\hat{\theta}))+2 p\left(\frac{n}{n-p-1}\right) \\
& =\mathrm{AIC}+\frac{2 p(p+1)}{n-p-1}
\end{aligned}
$$

whenever $n / p<40$. Anderson et al. (2001) believe that the use of $\mathrm{AIC}$ when $\mathrm{AIC}_{c}$ is more appropriate is a very common mistake when using the information-theoretic method.

Another modified criterion, $\mathrm{QAIC}_{c}$, has been developed for over-dispersed count data.

$$
\mathrm{QAIC}_{c}=-\left[\frac{2 \log (l(\widehat{\theta})}{\widehat{c}}\right]+2 p+\frac{2 p(p+1)}{n-p-1},
$$

where $\widehat{c}$ is the variance inflation factor (Burnham and Anderson 1998; Anderson et al. 2000, 2001).

Whichever model selection criterion is chosen, the same crition should be used for all candidate models in the set. The candidate model which minimizes the appropriate criterion is selected as the "best" model, and is used as the basis for data analysis and inference (Burnham and Anderson 1998).

One final note on information criteria. Burnham and Anderson (1998) also discuss information criteria that are "dimension consistent." One such criterion is Schwarz's Bayesian Information Criterion, BIC:

$$
\mathrm{BIC}=-2 \log (l(\widehat{\theta}))+p \log (n) .
$$

"Dimension consistent" criteria such as this one are based on the assumptions that an exactly "true" model exists, the "true" model is one of the candidate models, and the goal of model selection is to select the "true" model. Burnham and Anderson (1998) emphasize that these "dimension consistent" criteria are not estimates of the K-L distance, and are not recommended. 


\section{The Information-Theoretic Approach: Application}

An important tenet of the information-theoretic approach is selection of a set of a priori models based on careful thought about the relationships between the independent and dependent variables. Common ways to select the candidate models are using published literature and subject-matter knowledge in evaluating and selecting the independent variables in relation to the dependent variable in question. Once an a priori set of candidate models has been selected, and the appropriate information criterion has been calculated for all candidate models, the analysis question becomes one of model selection and inference on the selected model should be accomplished. Because $\mathrm{AIC}$ (as well as $\mathrm{AIC}_{c}$ and $\mathrm{QAIC}_{c}$ ) is a relative measure, Burnham and Anderson (1998) recommend calculating the AIC differences

$$
\Delta_{i}=\mathrm{AIC}_{i}-\min \mathrm{AIC}
$$

for each candidate model. This means that the "best" model will have $\Delta_{i}=0$. Burnham and Anderson (1998) also recommend that these differences, not the AIC values, be displayed in publications. These differences allow for easy model comparisons and rankings. The larger the $\Delta_{i}$, the less plausible the particular candidate model is "best" for data sets such as the one collected. Burnham and Anderson (1998) give some guidelines for interpretation of $\Delta_{i}$ values. If $\Delta_{i} \leq 2$, there is substantial support for the candidate model. If $4<\Delta_{i}<7$, there is considerably less support. If $\Delta_{i}>10$, there is no support for the candidate model. Based on these $\Delta_{i}$ values, the researcher selects the best and other supporting models, and identifies any models that are clearly not supported. It is recommended that any model with $\Delta_{i} \leq 4$ be given consideration (Burnham and Anderson 1998)

Burnham and Anderson (1998) and Anderson et al. (2000) also recommend making some additional calculations to better quantify the probability of each model being the "best." They show that a transformation of the $\Delta_{i}$ gives the likelihood of the model, given the data. This tranformation, recommended by Akaike as $\exp \left(-\frac{1}{2} \Delta_{i}\right)$, can be normalized so that the sum of the transformed $\Delta_{i}$ is 1 . These normalized values, called the Akaike weights, are calculated as

$$
w_{i}=\frac{\exp \left(-\frac{1}{2} \Delta_{i}\right)}{\sum_{r=1}^{R} \exp \left(-\frac{1}{2} \Delta_{r}\right)}
$$


where $r=1,2, \ldots, R$ and $R$ identifies the number of models in the candidate model set. The weight $w_{i}$ can be interpreted as the approximate probabilitiy that model $i$ is the "best" in the set of candidate models. Also, the relative likelihood of model $i$ versus model $j$ can be calculated as $w_{i} / w_{j}$ (Burnham and Anderson 1998; Anderson et al. 2000).

Once the selected model is chosen, inference may be undertaken. The analyst must remember that inference is conditional not only on the data set as usual, but also on the set of candidate models (Anderson et al. 2000). As a result of this conditioning on the set of the candidate models, the usual estimate of sampling variance must be adjusted by including a variance component to account for uncertainty in model selection. This adjustment ensures that estimates of precision are unconditional on the selected model, but are conditional on the candidate models in the set. Anderson et al. (2000) give an estimator of the unconditional variance for the parameter $\theta$ from the chosen model:

$$
\widehat{\operatorname{var}(\hat{\theta})}=\left[\sum_{i=1}^{R} w_{i} \sqrt{\left.\operatorname{var} \widehat{\left(\hat{\theta}_{i}\right.} \mid g_{i}\right)+\left(\hat{\theta}_{i}-\overline{\bar{\theta}}\right)^{2}}\right]^{2}
$$

where

$$
\widehat{\bar{\theta}}=\sum_{i=1}^{R} w_{i} \widehat{\theta}_{i} .
$$

This variance estimator, attributed to Buckland et al. (1997), includes the conditional sampling variance given model $g_{i}$ and a variance component for model uncertainty (Anderson et al. 2000). This unconditional variance can be used to construct the usual $95 \%$ confidence intervals for a parameter.

In some situations there will be no one clearly "best" model. Thus, instead of basing inference in a a single "best" model, inference can be based on an entire set of models by using a model-averaged estimator for a parameter $\theta$. The model-averaged estimator for $\theta$ is the weighted average previously shown:

$$
\widehat{\bar{\theta}}=\sum_{i=1}^{R} w_{i} \widehat{\theta}_{i} .
$$

A model-averaged estimator often has the advantages of better precision and reduced bias compared to the estimate of the parameter from a single "best" model (Anderson et al. 2000). Burnham and Anderson (1998) point out that when model-averaging, or simply calculating 
the estimated unconditional sampling variance for a parameter, the weights $w_{i}$ must be renormalized so that they sum to 1 for the subset of models that contain the parameters of interest.

\section{Case Study}

The information-theoretic approach to model selection will be illustrated using data from a study to determine the effects of landscape on bird species richness and abundance in riparian areas in southeastern Nebraska. This study was carried out by co-author Micah Perkins, a former graduate student in the School of Natural Resource Sciences at the University of Nebraska. The study was carried out in the summers of 1999 and 2000. In 1999, fifteen study sites in southeastern Nebraska were used and three additional sites were added in 2000 (Perkins 2001).

Study sites were linear strips of wooded riparian corridors $500 \mathrm{~m}$ in length and approximately $50 \mathrm{~m}$ wide. Each site was visited four times during each year, and bird species richness and abundance data were collected on each visit. Richness and abundance data were averaged over visit in each year. Local-scale measurements were made at each study site in each year. These included canopy cover and height, understory foliage volume, ground cover, stream measurements, and others. Landscape variables such as land cover, ratio of cropland to grassland, percentage of woody cover, and others were also measured on each site in each year. Details about data collection and a full list of measured variables may be found in Perkins (2001).

Dependent variables were woodland bird species abundance and richness, edge species abundance and richness, and abundance and presence of individual species. Independent variables were the local-scale and landscape measurements taken on each study site. Following a major tenet of the information-theoretic method, a set of a priori models was selected. These candidate models contained predictor variables thought to affect species group and individual species response variables. Thirty models were considered for each of the species group response variables. Anderson et al. (2001) recommend the number of candidate models $(R)$ be relatively small and suggest that situations with more models than observations $(R>n)$ be avoided. Because the Perkins (2001) data sets have $n=33$ observations, $R=30$ 
was the maximum number of models considered.

For the purpose of illustration of the information-theoretic approach, only the analyses for the response variable woodland species richness are presented. Analyses of the other response variables and discussion of the results are in Perkins (2001). For woodland species richness, 30 candidate models were considered. Each of these models has the form

$$
y_{i j}=\operatorname{year}_{i}+\beta_{0}+\beta_{1} \operatorname{predvar}_{1 i j}+\beta_{2} \operatorname{predvar}_{2 i j}+\ldots+\beta_{k} \operatorname{predvar}_{k i j}+\epsilon_{i j}
$$

where $i=1,2, j=1, \ldots, n_{i}\left(n_{1}=15, n_{2}=18\right)$ and $k$ is the total number of predictor variables for the model. The year effect was assumed to be random. This means that for each candidate model, there are $k$ predictor variable parameters, one intercept, and two variance components estimated. This gives a total of $p=k+3$ for the penalty term in the appropriate information criterion. Because of the small sample size, $\mathrm{AIC}_{c}$ was used. The independent variables (Table 1) were used in 30 models, shown with their $\mathrm{AIC}_{c}, \Delta_{i}$ values, and Akaike weights $w_{i}$ (Table 2). The models are shown in increasing order of $\mathrm{AIC}_{c}$ values; however, it doesn't matter in which order the models are fit, nor does the order of variables matter.

The first five models in Table 2 have $\Delta_{i}<4$, and are given consideration. Recall that $w_{i}$ is interpreted as the approximate probability that model $i$ is the K-L "best." The $w_{i}$ for models in which a particular variable appears may be summed to provide an indication of the importance of that variable (Anderson et al. 2000). The variable p500 (percent woody cover out to $500 \mathrm{~m}$ ) appears in 14 of the 30 models and has a sum of $w_{i}=0.77803$. This variable also appears in four out of the five top models. The next most important variable is GCSoil (ground cover percentage in soil), with $\sum w_{i}=0.46277$.

Anderson et al. (2000) also point out that $w_{i} / w_{j}$ is the relative likelihood of model $i$ versus model $j$. Comparing the first and second models gives $w_{1} / w_{2}=0.37123 / 0.21418=1.733$. This implies that model 1 is not clearly superior to model 2, and rather than ignore model selection uncertainty, the model-averaged estimates of the effects and their unconditional standard errors were calculated. The 14 models containing the p500 variable, the individual model estimates of the p500 regression coefficient $\left(\widehat{\beta}_{\mathrm{p} 500}\right)$, conditional standard errors $\left(\operatorname{se}\left(\widehat{\beta_{\mathrm{p} 500}} \mid g_{i}\right)\right)$, and re-scaled $w_{i}$, are given in Table 3 . The first step is to find the modelaveraged estimate of the effect of p500. This is simply the weighted average of the 14 


\section{Applied Statistics in Agriculture}

individual estimates of the p500 effect.

$$
\widehat{\bar{\beta}}_{\mathrm{p} 500}=\sum_{i=1}^{14} w_{i} \widehat{\beta}_{\mathrm{p} 500 i}=(0.47713)(0.04350)+\ldots+(0.00000)(0.07609)=0.05773
$$

The next step is to calculate the unconditional standard error for the p500 effect. The unconditional variance for the p500 effect is

$$
\begin{aligned}
\operatorname{var}\left(\widehat{\widehat{\beta}}_{\mathrm{p} 500}\right)= & {\left[\sum_{i=1}^{14} w_{i} \sqrt{\operatorname{var}\left(\widehat{\widehat{\beta}}_{\mathrm{p} 500 i} \mid g_{i}\right)+\left(\widehat{\beta}_{\mathrm{p} 500 i}-\widehat{\bar{\beta}}_{\mathrm{p} 500}\right)^{2}}\right]^{2} } \\
= & {\left[(0.47713) \sqrt{(0.01400)^{2}+(0.04350-0.05773)^{2}}\right.} \\
& +(0.18453) \sqrt{(0.01578)^{2}+(0.07859-0.05773)^{2}}+\ldots \\
& \left.+(0.00000) \sqrt{(0.01635)^{2}+(0.06509-0.05773)^{2}}\right]^{2} \\
= & 0.00045484
\end{aligned}
$$

The unconditional standard error for the p500 effect is therefore $\sqrt{0.00045484}=0.021327$. An approximate $95 \%$ confidence interval for the effect of p500 on woodland species richness would then be $\widehat{\bar{\beta}}_{\mathrm{p} 500} \pm 2 \mathrm{se}\left(\widehat{\widehat{\beta}}_{\mathrm{p} 500}\right)$, or $0.05773 \pm 0.04265$.

Model-averaged estimates and unconditional standard errors could be found similarly for all of the predictor variables in the best models.

To compare the information-theoretic approach to the standard approach, the stepwise regression method was also used to determine which subset of the variables in Table 1 best explain the variation in woodland species richness. However, due to the limitations of PROC REG, the random year effect included in the 30 candidate models was not included in the stepwise model. The first three variables selected by the stepwise procedure were p500, GCSoil and GCLitter, the same variables in the minimum $\mathrm{AIC}_{c}$ model. The stepwise procedure went on to select a1000, C3, ufv12, and a500 as well. During the course of the procedure, p500 and GCSoil were removed from the model. The information-theoretic approach selected these two variables as the most important, with $\sum w_{i}=0.77803$ and 0.46277, respectively. So, while the initial variables chosen by the stepwise procedure are the same as those selected by the information-theoretic approach, the results from the two approaches are quite different. 


\section{Conclusion}

The information-theoretic approach to model selection is becoming more widely used in the wildlife science literature. The advantages of the approach include its ability to compare non-nested models and its emphasis on a priori thought rather than data dredging. There have been some criticisms of the method, however. Guthery et al. (2001) point out that likelihood based methods are by definition parametric. If an incorrect probability distribution is assumed for the data, the inference may not be valid. Guthery et al. (2001) also worry that the current rote approach to significance testing will simply be replaced by a rote calculation of information criteria.

There are many situations in which the information-theoretic approach to model selection is inappropriate. It is not meant to replace hypothesis testing in designed experiments. In formal experimentation, specific predictor variables are chosen and their effects can be validly assessed by testing specific a priori null and alternative hypotheses. In observational studies, however, analyses tend to focus on model selection (i.e., which of the myriad variables observed are useful in explaining the response). In this situation, the information-theoretic method assists the scientist in model selection while avoiding significance tests for clearly false null hypotheses. Moreover, the method is not difficult to apply and can be approached using standard statistical software.

\section{Acknowledgements}

This is Journal Paper No. 13921 of the Nebraska Argicultural Research Division, University of Nebraska at Lincoln.

\section{References}

[1] Anderson, D. R., Burnham, K. P., and Thompson, W. L. (2000) "Null Hypothesis Testing: Problems, Prevalence, and an Alternative," Journal of Wildlife Management, 64, 912-923.

[2] Anderson, D. R., Link, W. A., Johnson, D. H., and Burnham, K. P. (2001) "Suggestions for Presenting the Results of Data Analyses," Journal of Wildlife Management, 65, 373- 
378.

[3] Buckland, S. T., Burnham, K. P. and Augustin, N. H. (1997) "Model Selection: An Integral Part of Inference," Biometrics, 53, 603-618.

[4] Burnham, K. P. and Anderson, D. R. (1992) "Data-Based Selection of an Appropriate Biological Model: The Key to Modern Data Analysis," in Wildlife 2001: Populations (D. R. McCullough and R. H. Barrett, editors), Elsevier Applied Science, London, pages 16-30.

[5] Burnham, K. P. and Anderson, D. R. (1998) Model Selection and Inference: A Practical Information-Theoretic Approach, Springer, New York.

[6] Cherry, W. (1998) "Statistical Tests in Publications of The Wildlife Society," Wildlife Society Bulletin, 26, 947-953.

[7] Guthery, F. S., Lusk, J. J. and Peterson, M. J. (2001) "The Fall of the Null Hypothesis: Liabilities and Opportunities," Journal of Wildlife Management, 65, 379-384.

[8] Johnson, D. H. (1999) "The Insignificance of Statistical Significance Testing," Journal of Wildlife Management, 63, 763-772.

[9] Perkins, M. W. (2001) Effects of Woody Cover in the Landscape on Riparian Avifauna and a Preliminary Nesting Assessment, thesis, University of Nebraska-Lincoln, Lincoln, Nebraska. 
Table 1: Woodland Species Richness: Abbreviations for Independent Variables

\begin{tabular}{ll} 
Abbreviation & Description \\
\hline p500 & percent woody cover out to $500 \mathrm{~m}$ \\
p1000 & percent woody cover out to $1000 \mathrm{~m}$ \\
p2000 & percent woody cover out to $2000 \mathrm{~m}$ \\
a500 & area weighted shape index out to $500 \mathrm{~m}$ \\
a1000 & area weighted shape index out to $1000 \mathrm{~m}$ \\
a2000 & area weighted shape index out to $2000 \mathrm{~m}$ \\
areaha & area of study site \\
GCSoil & ground cover percentage in soil \\
GCLitter & ground cover percentage in litter \\
GCGrass & ground cover percentage in grass \\
GCWoody & ground cover percentage in woody growth \\
GCForb & ground cover percentage in forbs \\
ufv03 & understory foliage volume $0-0.3 \mathrm{~m}$ \\
ufv031 & understory foliage volume $0.3-1 \mathrm{~m}$ \\
ufv12 & understory foliage volume $1-2 \mathrm{~m}$ \\
ufv23 & understory foliage volume $2-3 \mathrm{~m}$ \\
dsd & deciduous shrub density \\
ch & canopy height \\
snag & number of standing dead trees \\
r500 & ratio of crop land to grass land out to $500 \mathrm{~m}$ \\
C1 & trees $3-8 \mathrm{~cm}$ dbh \\
C2 & trees $8-15 \mathrm{~cm}$ dbh \\
C3 & trees $15-23 \mathrm{~cm}$ dbh \\
C4 & trees $23-38 \mathrm{~cm}$ dbh \\
C5 & trees $38-53 \mathrm{~cm}$ dbh \\
C6 & $53 \mathrm{~cm}$ dbh \\
&
\end{tabular}


Table 2: Woodland Species Richness: Candidate Models, $\mathrm{AIC}_{c}, \Delta_{i}$, and $w_{i}$

\begin{tabular}{lccc} 
Independent Variables & $\mathrm{AIC}_{c}$ & $\Delta_{i}$ & $w_{i}$ \\
\hline p500, GCSoil, GCLitter & 80.9 & 0 & 0.37123 \\
ufv03, ufv031, ufv12, ufv23 & 82.0 & 1.1 & 0.21418 \\
p500, GCGrass, GCWoody, ufv03 & 82.8 & 1.9 & 0.14357 \\
p500, GCGrass, GCWoody & 83.2 & 2.3 & 0.11754 \\
p500, GCSoil & 83.7 & 2.8 & 0.09154 \\
p500, GCLitter & 87.3 & 6.4 & 0.01513 \\
p500, GCWoody & 87.3 & 6.4 & 0.01513 \\
p500, uvf03, ufv031 & 87.7 & 6.8 & 0.01239 \\
p500, GCGrass & 88.2 & 7.3 & 0.00965 \\
ufv03, ufv031 & 88.8 & 7.9 & 0.00715 \\
p500, ufv03 & 92.5 & 11.6 & 0.00112 \\
ufv03, GCGrass & 93.8 & 12.9 & 0.00059 \\
p500, GCForb & 94.2 & 13.3 & 0.00048 \\
p500, areaha & 96.2 & 15.3 & 0.00018 \\
p500, a500 & 98.5 & 17.6 & 0.00006 \\
p1000, areaha & 99.6 & 18.7 & 0.00003 \\
p1000, a1000 & 101.2 & 20.3 & 0.00001 \\
p500, a500, areaha & 101.2 & 20.3 & 0.00001 \\
p1000, a1000, areaha & 104.1 & 23.2 & 0.00000 \\
p2000, areaha & 106.0 & 25.1 & 0.00000 \\
p500, r500 & 107.0 & 26.1 & 0.00000 \\
p2000, a2000 & 107.7 & 26.8 & 0.00000 \\
ch, snag & 108.0 & 27.1 & 0.00000 \\
ufv03, ufv031, ufv12, ufv23, dsd, ch & 108.7 & 27.8 & 0.00000 \\
dsd, ufv03, ufv031 & 108.7 & 27.8 & 0.00000 \\
p2000, a2000, areaha & 109.3 & 28.4 & 0.00000 \\
ch, snag, dsd, ufv03, ufv031 & 115.4 & 34.5 & 0.00000 \\
C4, C5, C6 & 119.5 & 38.6 & 0.00000 \\
C4, C5, C6, g500 & 122.5 & 41.6 & 0.00000 \\
C1, C2, C3, C4, C5, C6 & 141.2 & 60.3 & 0.00000 \\
& & &
\end{tabular}


Table 3: Woodland Species Richness: 14 Models containing p500, estimates of $\widehat{\beta}_{\mathrm{p} 500}$, $\operatorname{se}\left(\widehat{\beta_{\mathrm{p} 500}} \mid g_{i}\right)$ and re-scaled $w_{i}$

\begin{tabular}{lccc} 
Model & $\widehat{\beta}_{\mathrm{p} 500}$ & $\mathrm{se}\left(\widehat{\widehat{\beta}_{\mathrm{p} 500}} \mid g_{i}\right)$ & $w_{i}$ \\
\hline p500, GCSoil, GCLitter & 0.04350 & 0.01400 & 0.47713 \\
p500, GCGrass, GCWoody, ufv03 & 0.07859 & 0.01578 & 0.18453 \\
p500, GCGrass, GCWoody & 0.07807 & 0.01543 & 0.15108 \\
p500, GCSoil & 0.05356 & 0.01328 & 0.11766 \\
p500, GCLitter & 0.04671 & 0.01511 & 0.01945 \\
p500, GCWoody & 0.08711 & 0.01658 & 0.01945 \\
p500, ufv03, ufv031 & 0.05375 & 0.01523 & 0.01592 \\
p500, GCGrass & 0.06108 & 0.01311 & 0.01240 \\
p500, ufv03 & 0.06845 & 0.01529 & 0.00144 \\
p500, GCForb & 0.06678 & 0.01456 & 0.00062 \\
p500, areaha & 0.05933 & 0.01577 & 0.00023 \\
p500, a500 & 0.06378 & 0.01526 & 0.00007 \\
p500, a500, areaha & 0.05900 & 0.01611 & 0.00002 \\
p500, r500 & 0.06509 & 0.01635 & 0.00000
\end{tabular}

